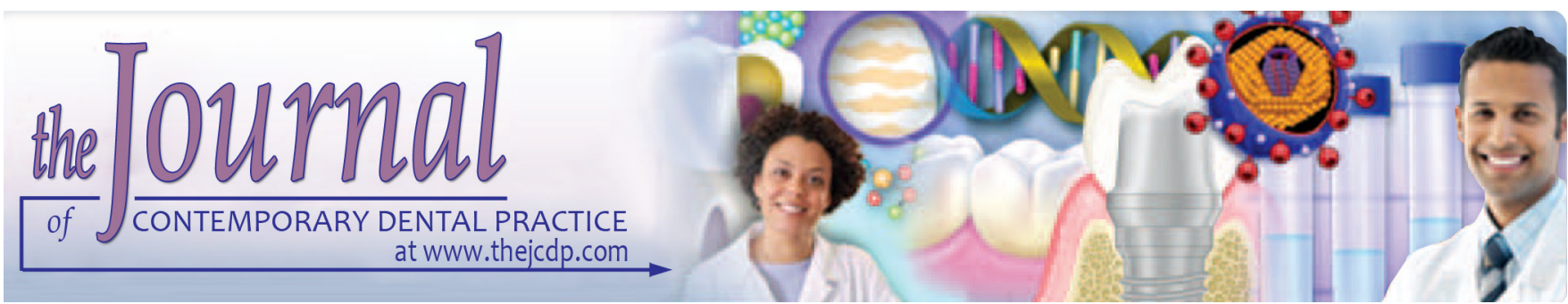

\title{
Efficacy of Ozonised Water and $0.2 \%$ Chlorhexidine Gluconate in the Management of Chronic Periodontitis when Used as an Irrigant in Conjugation with Phase I Therapy
}

\author{
${ }^{1}$ Amritpal Kaur, ${ }^{2}$ Shaeesta K Bhavikatti, ${ }^{3}$ Sitansu S Das, ${ }^{4}$ Sagar Khanna, ${ }^{5}$ Meena Jain, ${ }^{6}$ Avineet Kaur
}

\begin{abstract}
Aim: To evaluate the efficacy of irrigation of periodontal pockets by using ozonated water and $0.2 \%$ chlorhexidine $(\mathrm{CHX})$ gluconate as adjuncts to scaling and root planing in the management of chronic periodontitis.

Materials and methods: For the present study, 20 patients in the age group of 30-60 years, suffering from chronic periodontitis presenting with at least one site with an almost similar pocket depth of 4-6 $\mathrm{mm}$ in both the quadrants of maxillary arch was taken. Irrigation was done after 2 weeks of scaling and root planning on the same day with ozonated water and $0.2 \%$ chlorhexidine gluconate for two and half minutes. The clinical parameters like gingival index, probing pocket depth (PPD) and clinical attachment level (CAL) were recorded for both the groups at the baseline visit.They were subsequently recalled after 4 weeks and 3 months interval from the baseline visit. Data thus collected was compiled and put to statistical analysis.
\end{abstract}

\footnotetext{
${ }^{1}$ Department of Periodontology, Surendera Dental College, Hospital and Research Institute, Sriganganagar, Rajasthan, India

${ }^{2}$ Division of Periodontics and Community Dental Sciences, College of Dentistry, King Khalid University, Abha, Kingdom of Saudi Arabia

${ }^{3}$ Department of Prosthodontics, Institute of Dental Sciences, Siksha O Anusandhan Deemed to be University, Bhubaneswar, Odisha, India

${ }^{4}$ Department of Conservative Dentistry and Endodontics, Mansarovar Dental College, Bhopal, Madhya Pradesh, India

${ }^{5}$ Department of Public Health Dentistry, Manav Rachna Dental College, Faridabad, Haryana, India

${ }^{6}$ Department of Periodontolgy and Oral Implantology, Maharishi Markandeshwar College of Dental Sciences and Research, Mullana, Haryana India
}

Corresponding Author: Shaeesta K Bhavikatti, Division of Periodontics and Community Dental Sciences, College of Dentistry, King Khalid University, Abha, Kingdom of Saudi Arabia, Phone: +966550654934, e-mail: sbhavhkatti@kku. edu.sa
Results: The present study showed significant results in both the groups with regards to the improvement in the clinical parameters. When comparison was made between the two groups, ozonated water showed slightly better improvement than the chlorhexidine group. However, a statistically significant difference was seen only with Plaque score.

Conclusion: Subgingival irrigation with ozonized water is beneficial than present conventional therapeutic modalities. Ozonated water restricts the formation of dental plaque and reduces the number of subgingival pathogens thereby treating periodontal diseases.

Clinical significance: Ozone therapy is proving to be a new useful treatment modality which offers great benefits to the patients. The strong antimicrobial power of ozone, along with its ability to stimulate the circulatory system and modulate the immune response, makes it a remedial agent of choice in the treatment of various infectious oral diseases. The study was conducted to justify the routine use of ozone as a treatment modality in effective management of periodontal diseases.

Keywords: Chlorhexidine gluconate, Chronic periodontitis, Irrigation, Ozone.

How to cite this article: Kaur A, Bhavikatti SK, Das SS, Khanna S, Jain M, Kaur A. Efficacy of Ozonised Water and $0.2 \%$ Chlorhexidine Gluconate in the Management of Chronic Periodontitis when Used as an Irrigant in Conjugation with Phase I Therapy. The Journal of Contemporary Dental Practice, 2019;20(3):318-323.

Source of support: Nil

Conflict of interest: None

\section{INTRODUCTION}

The accumulation and maturation of dental plaque at the gingival margin are widely recognized as the primary etiologic factor in the development of periodontal disease. ${ }^{1}$ Removal of matured dental plaque and controlling its further accumulation as well as maturation is an important part of periodontal therapy. Most widely acknowledged method for supragingival plaque control is mechanical tooth brushing but it does not appear to act against deep subgingival plaque, ${ }^{2}$ that 
may be the reasons that has encouraged the use of various systemic or local chemotherapeutic agents as adjuncts to mechanical therapy. The systemic antibacterial agents achieve a low concentration in the periodontal pockets and have certain unwanted effects, ${ }^{3}$ whereas local antimicrobial agents achieve a higher concentration in the periodontal site at very low concentration. The bisbiguanide salt, chlorhexidine gluconate ( $\mathrm{CHX)} \mathrm{considered}$ the gold standard has a broad antimicrobial spectrum ${ }^{4}$ conceptually, used as supra- and sub-gingival irrigation by therapists and patients to help suppress bacterial etiologic agents.

An alternative approach to this usual treatment in inhibition of subgingival bacteria is to reduce their growth by changing the subgingival environment which is highly anaerobic with low oxygen tension. Various treatment modalities such as hyperbaric oxygenation, molecular oxygen, and hydrogen peroxide have been applied with the concept of oxygenating the periodontal tissues. $^{5}$

Currently, ozone in gaseous or aqueous form is being discussed in dentistry as a possible alternative antiseptic agent. The potent antimicrobial power of ozone without the development of drug resistance along with its capacity to stimulate the circulatory system and modulate the immune response makes it a therapeutic agent of choice. ${ }^{6}$

The purpose of the present study was to compare the efficacy of subgingival irrigation with ozonated water to $0.2 \%$ chlorhexidine ( $\mathrm{CHX})$ as adjuncts to scaling and root planning in chronic periodontitis.

\section{MATERIALS AND METHODS}

Twenty patients of chronic periodontitis in the age group of 30-60 years of both the sexes were selected from the outpatient department of Periodontology and Oral Implantology, Guru Nanak Dev Dental College and Research Institute, Sunam.

Pregnant or lactating women, patients suffering from any known systemic disease, the patient who underwent any surgery or had received any antibiotic therapy, chemotherapeutic mouth rinses and oral irrigation 3 months ibefore the start of the study, were excluded from the study. A randomized single-blind split-mouth design was used. All patients were informed of the benefits and risks of the study and consent was taken. After education and motivation, each patient was put on mechanical plaque control measures followed by full mouth supragingival scaling.

All patients were recalled four weeks after completion of supragingival scaling which formed the baseline visit. Patients who returned with the PPD in the range of 4-6 $\mathrm{mm}$ at least at one of the sites, in each quadrant of the maxillary arch were selected, thus making a total of 40 sites. At baseline, the clinical parameters like gingival index, PPD and clinical attachment level (CAL) were recorded.

Subgingival scaling of the 40 selected sites was done and plaque score was brought to zero. Randomly selected ten patients received $0.2 \% \mathrm{CHX}$ irrigation in selected sites (10 sites) on the left side and ozonated water irrigation in selected sites (10 sites) on the right side of the maxillary arch. Other ten patients received ozonated water irrigation in selected sites (10 sites) on the left side and $0.2 \%$ CHX irrigation in selected sites (10 sites) on the right side.

The study was divided into two groups:

Group I: Where 20 sites received chlorhexidine irrigation Group II: Where 20 sites received ozonated water irrigation.

The irrigation with ozonated water was done with an irrigation device, 'Kent ozone Dental Jet' (Fig. 1). The sites on the contralateral side were irrigated with a commercially available $0.2 \% \mathrm{CHX}$ solution delivered via a Dentos Waterpik (Fig. 2). Irrigation was done for two and a half minutes with same pressure setting, and excess irrigant was continuously aspirated. After irrigation, the patients were instructed to brush twice daily by modified Stillman's technique using a standard toothbrush and toothpaste provided to them. They were instructed not to use any mouth rinse.

Patients were recalled after a week to record the Plaque score. They were subsequently recalled after 4 weeks and 3 months interval from the baseline visit to record the Plaque score (Quigley- Hein, and Elliot index), Gingival score (Loe and Silness index 1963), PPD and relative CAL.

Data were analyzed using statistical package for the social sciences (SPSS) version 13 (SPSS, Chicago, IL, USA). Frequency and percentage distributions were calculated. The student's $t$-test was used to find the statistical difference in the means of $\mathrm{CHX}$ and ozonated water at $p$ value $<0.05$.

\section{RESULTS}

Values of mean Plaque score for inter-group comparison were noted at various time interval as shown in Table 1. The difference between the means was statistically significant at 4 weeks and 3 months ( $p$ value $<0.05)$ and non- significant ( $p$ value $>0.05$ ) at 1 week but the plaque increment on the sites treated with ozonated water was less as compared to $\mathrm{CHX}$. Figures 3 and 4 depict Plaque score at 3 months in groups I and II respectively.

Table 2 depicts the inter-group comparison of the mean gingival score which was statistically nonsignificant ( $p$ value $>0.05)$ at baseline, 4 weeks and 3 months. But the percentage reduction of gingival score at 4 weeks and 3 months from baseline was slightly higher in the ozonated water (30.0\% and 59.1\%) as compared to CHX $(30.0 \%$ and $50.8 \%)$. 


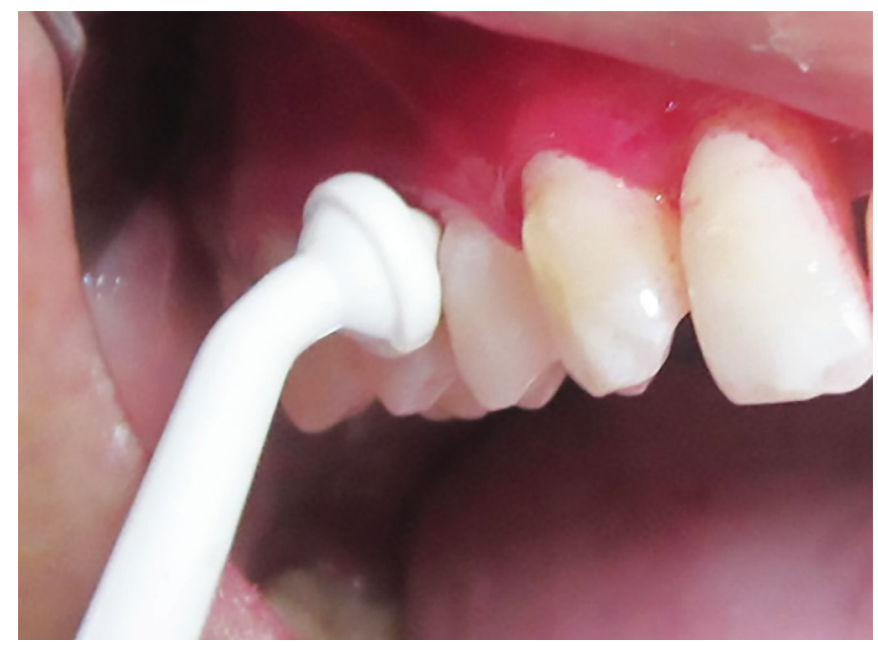

Fig. 1. Ozonated water irrigation on left side

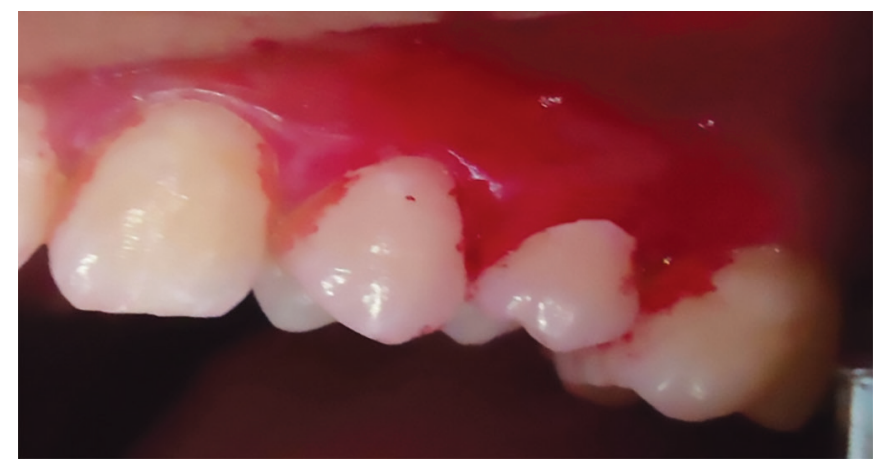

Fig. 3: Plaque score at 3 months in group I (chlorhexidine)

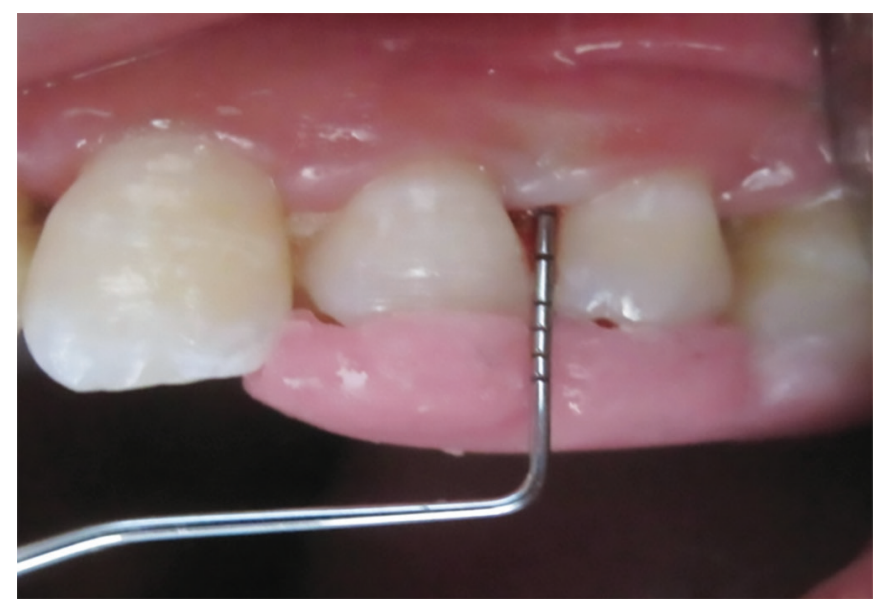

Fig. 5: Probing pocket depth and clinical attachment level at 3 months in group I (Chlorhexidine)

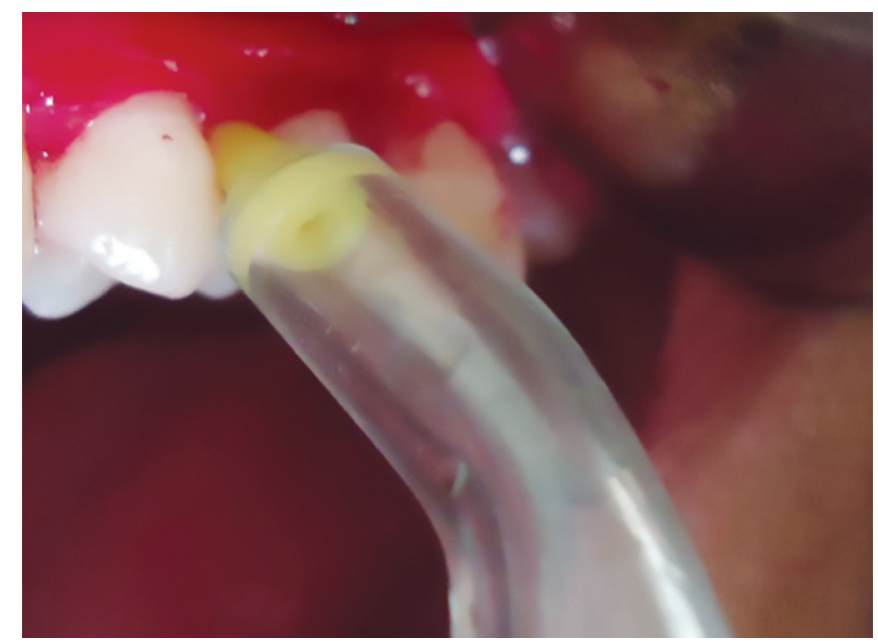

Fig. 2: Chlorhexidine irrigation on right side

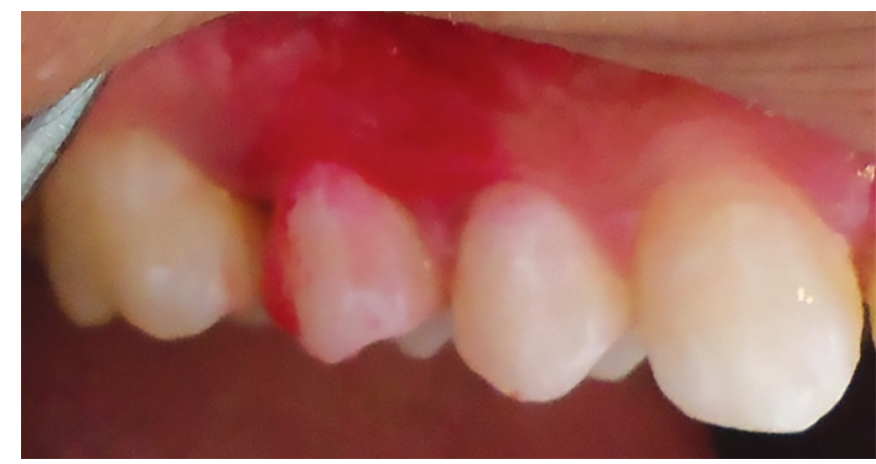

Fig. 4: Plaque score at 3 months in group II (ozonated water)

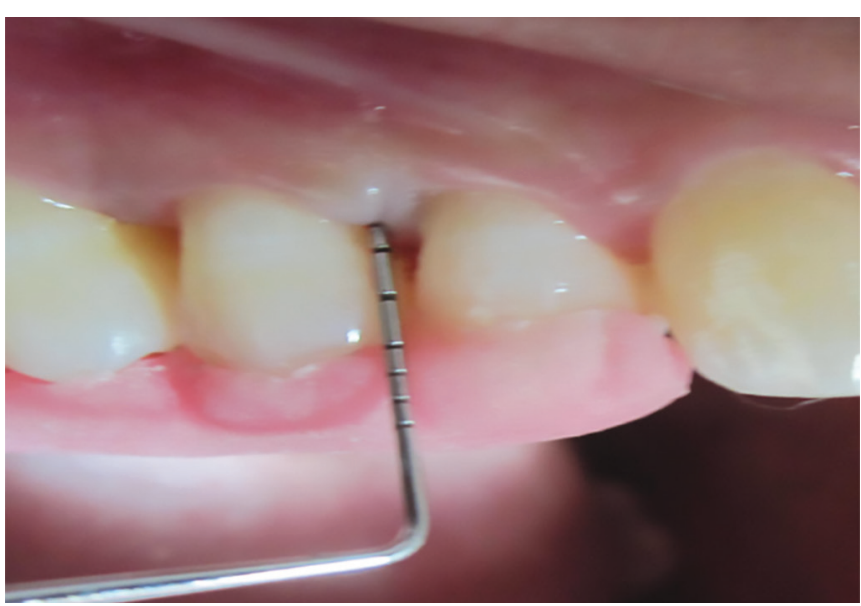

Fig. 6: Probing pocket depth and clinical attachment level at 3 months in group II (Ozonated water)

Table 1: Inter-group comparison of the mean Plaque score at baseline, 1 week, 4 weeks and 3 months

\begin{tabular}{llllll}
\hline & Chlor-hexidine gluconate & ozonated water & $T$ & $p$ value & Significance \\
\hline PI 1 week & 2.00 & 1.675 & 1.748 & 0.089 & NS \\
PI 4 weeks & 1.90 & 1.475 & 2.494 & 0.017 & $\mathrm{~S}$ \\
PI 3 months & 2.25 & 1.70 & 2.463 & 0.018 & $\mathrm{~S}$ \\
\hline
\end{tabular}

Inter-group comparison of the mean plaque score at baseline, 1 week, 4 weeks and 3 months

Table 2: Inter-group comparison of the mean Gingival score at baseline, 4 weeks and 3 months

\begin{tabular}{llllll}
\hline & Chlor-hexidine gluconate & Ozonated water & $T$ & $p$ value & Significance \\
\hline GI baseline & 2.25 & 2.40 & -1.000 & 0.324 & NS \\
GI 4 weeks & 1.60 & 1.75 & -0.679 & 0.501 & NS \\
GI 3 months & 1.15 & 1.05 & 0.420 & 0.677 & NS \\
\hline
\end{tabular}

Inter-group comparison of the mean gingival score at baseline, 4 weeks and 3 months 
Table 3 depicts the inter-group comparison of the mean PPD which was statistically nonsignificant ( $p$-value $>0.05$ ) at baseline, 4 weeks and 3 months. But the percentage reduction in PPD at 4 weeks and 3 months from baseline was slightly better in ozonated water $(14.5 \%$ and $34.5 \%)$ as compared to $\mathrm{CHX}(12.0 \%$ and $27.0 \%$ ) as shown in Table 4. Figures 5 and 6 shows the Probing Pocket Depth (PPD) and clinical Attachment Level at 3 months at 3 months in groups I and II respectively.

The statistical analysis of the inter-group comparison of the mean Clinical Attachment Gain was statistically nonsignificant ( $p$ value $>0.05$ ) at baseline, 4 weeks and 3 months (Table 5). The percentage gain in clinical attachment level from baseline to 3 months for ozonated water $(11.5 \%)$ was slightly better than chlorhexidine $(9.5 \%)$ as shown in Table 4.

\section{DISCUSSION}

With the increasing awareness of microbial etiology of the periodontal disease, a more direct approach using antimicrobial agents as an adjunct to mechanical therapy has become an integral part of the therapeutic armamentarium. ${ }^{7}$

Antimicrobial agents can be used both systemically and locally. The systemic administration of antibacterial agents achieves considerable success, but due to their low concentration in the periodontal pockets and certain unwanted effects, the concept of local delivery of antibacterial agents into periodontal pockets was developed by Goodson et al. ${ }^{8}$

Local antimicrobial therapy in periodontitis involves direct delivery of an antimicrobial agent(s) into subgingival sites, thereby reducing the systemic effect as compared to systemic therapy. Local delivery of antimicrobial agents can be in the form of mouth rinses, gels, chips, ointments, and pocket irrigation. Subgingival irrigation is the most widespread means of applying antimicrobials subgingivally whether professionally or by the patient. ${ }^{9}$

Chlorhexidine gluconate, a cationic bisbiguanide, has a broadspectrum antibacterial effect by virtue of its high intra-oral substantivity. Many studies have shown the valuable effects of irrigating pockets with chlorhexidine, but the use of chlorhexidine is burdened by some side effects that could affect patient compliance. ${ }^{1,10,11}$

The use of ozone is justified as a new option of irrigating agent with antimicrobial action without developing resistance which results from oxidation of microbial cellular components. ${ }^{12}$ however ozone becomes highly reactive and unstable when it comes in contact with water thereby resulting in its rapid disintegration resulting in generation of hydroxyl radicals, which are among the most reactive oxidizing species. ${ }^{5}$ The

Table 3: Intergroup comparison of mean probing pocket depth at baseline, 4 weeks and 3 months

\begin{tabular}{llllll}
\hline & Chlor-hexidine gluconate & Ozona-ted water & $T$ & $p$ value & Signi-ficance \\
\hline PPD baseline & 4.25 & 4.50 & -1.648 & 0.108 & NS \\
PPD 4 weeks & 3.75 & 3.85 & -0.456 & 0.651 & NS \\
PPD 3 months & 3.10 & 2.95 & 0.588 & 0.560 & NS \\
\hline
\end{tabular}

Inter-group comparison of mean Probing Pocket Depth at baseline, 4 weeks and 3 months

Table 4: Intra-group comparison of changes in mean probing pocket depth at baseline, 4 weeks and 3 months

\begin{tabular}{|c|c|c|c|c|c|c|}
\hline Groups & Difference & Mean difference & Std. deviation & $\begin{array}{l}\text { Percentage (\%) } \\
\text { improvement }\end{array}$ & $t$ & $p$ \\
\hline \multirow[t]{3}{*}{ Chlorhexidine Gluconate } & Baseline -4 weeks & 0.500 & 0.513 & 12.0 & 4.359 & $<.001$ \\
\hline & Baseline -3 months & 1.150 & 0.813 & 27.0 & 6.328 & $<.001$ \\
\hline & 4 weeks- 3 months & 0.650 & 0.587 & 17.4 & 4.951 & $<.001$ \\
\hline \multirow[t]{3}{*}{ Ozonated Water } & Baseline - 4 weeks & 0.650 & 0.489 & 14.5 & 5.940 & $<.001$ \\
\hline & Baseline -3 months & 1.550 & 0.686 & 34.5 & 10.100 & $<.001$ \\
\hline & 4 weeks- 3 months & 0.900 & 0.447 & 23.8 & 9.000 & $<.001$ \\
\hline
\end{tabular}

Intra-group comparison of changes in mean probing pocket depth at baseline, 4 weeks and 3 months

Table 5: Inter-group comparison of the mean clinical attachment level at 4 weeks and 3 months

\begin{tabular}{llllll}
\hline & Chlorhexidine gluconate & Ozonated water & $T$ & $p$ value & Significance \\
\hline CAL baseline & 7.80 & 7.90 & -0.291 & 0.773 & NS \\
CAL 4 weeks & 7.60 & 7.80 & -0.551 & 0.585 & NS \\
Clinical attachment gain 4 weeks & 0.20 & 0.10 & 0.872 & 0.389 & NS \\
CAL 3 months & 7.05 & 7.00 & 0.142 & 0.888 & NS \\
Clinical attachment gain 3 months & 0.75 & 0.90 & -1.064 & 0.294 & NS \\
\hline
\end{tabular}

Inter-group comparison of the mean Clinical Attachment Level at 4 weeks and 3 months 
aqueous ozone reveals a high level of biocompatibility to fibroblasts, cementoblasts, and epithelial cells. ${ }^{13}$ Other biological actions of ozone include immunostimulation, immunomodulation, anti-inflammatory action, biosynthetic, bioenergetic, antihypoxic, analgesic, and hemostatic actions etc. ${ }^{14}$

The disinfectants can inactivate bacteria by two methods: physicochemical damage to cell surface components followed by damage to intracellular constituents and impairment of their function or direct impairment of intracellular functions without damage to surface structures. Both chlorhexidine and ozone mechanize by disrupting the cell wall, thereby damaging the cellular components. ${ }^{15}$

Various studies have reported encouraging effects with chlorhexidine and ozone irrigation. ${ }^{1,5,16,17}$ The present study was conducted with the objective to compare the effect of $0.2 \%$ chlorhexidine and ozone irrigation in the treatment of chronic periodontitis.

In the present study, 20 patients of both the sexes in the age range of 30 to 60 years were recruited. A split-mouth design was used to remove all the components related to differences between subjects by making within-patient rather than between-patient comparisons, error variance of the experiment was reduced.

To eliminate the cross-across or spillover effects, the sites selected in the maxillary arch only and were with respect to 6 teeth and excess irrigant was continuously aspirated. $^{18,19}$

In the present study, the effect of single irrigation was evaluated which is in accordance with the studies by Taggart et al., ${ }^{20}$ Christersson et al., ${ }^{21}$ Stabholz et al., ${ }^{22}$ who observed significant improvement with single irrigation. The subgingival scaling and root planning were carried out before the irrigation and plaque score was brought to zero at baseline. This was similar to a study by Kalaga et al. ${ }^{23}$ who evaluated the effect of chlorhexidine spray and a mouth rinse from a zero baseline value.

In the present study, when the comparison was made between the two groups, ozonated water showed slightly better improvement than the chlorhexidine. However, a statistically significant difference was seen only with Plaque score. Ramzy et al. ${ }^{24}$ found a significant improvement in Plaque score treated by scaling and root planing along with ozone application. The plaque increment on the sites treated with ozonated water was less as compared to that of chlorhexidine and this is in accordance with Kshitish and Laxman. ${ }^{5}$ This could be due to better antimicrobial action of ozonated water as observed by Huth et al. in $2011^{15}$ who found a significant reduction in periodontal pathogens on irrigation with ozone as compared to $0.2 \%$ CHX. Nagayoshi et al. ${ }^{25}$ found that ozonated water had strong bactericidal activity in a biofilm and also inhibited the accumulation of plaque in vitro.

The percentage reduction of Gingival score at 4 weeks and 3 months was slightly higher in the ozone as compared to chlorhexidine. Irrigation of the gingival tissue results in an alteration in the composition of plaque thereby resulting in reduced inflammation as well as resulting in healthier gingival tissue. These findings are in accordance with Kshitish and Laxman. ${ }^{5}$ Ramzy et al. ${ }^{24}$ found a significant improvement in PPD, Plaque score, Gingival score, and bacterial count with ozone application. According to Huth et al., ${ }^{13}$ aqueous ozone inhibited NF-kB activity in periodontally damaged teeth which suggests anti-inflammatory effects.

The percentage reduction in PPD was slightly better in ozone $(14.5 \%$ and $34.5 \%)$ as compared to chlorhexidine $(12.0 \%$ and $27.0 \%)$. This is in accordance with Dodwad et al. ${ }^{17}$ who observed a higher percentage of PPD reduction using ozone compared to chlorhexidine and povidone iodine. The similar results were also observed by Ramzy et al. ${ }^{24}$ This can be attributed to better antimicrobial action against $P$. gingivalis, Parvimonas Micra, Tannerella forsythia on irrigation with ozone compared to chlorhexidine as observed by Huth et al. ${ }^{15}$

The percentage gain in CAL from baseline to 3 months for ozonated water $(11.5 \%)$ was slightly better than chlorhexidine $(9.5 \%)$ which can be attributed to better antimicrobial action of ozonated water and higher level of biocompatibility to fibroblasts and epithelial cells which helps in healing as observed by Huth et al. ${ }^{26}$ On the contrary, Bassetti and Kallenberger ${ }^{27}$ observed impairment of wound healing and Cline and Layman ${ }^{28}$ reported reduced attachment of fibroblasts to root surfaces with chlorhexidine.

Chlorhexidine is considered the gold standard, but it has been discouraged as a form of prolonged treatment due to its certain side effects. Ozone is currently being discussed as a possible alternative agent as it is free from side effects of chlorhexidine. In the present study, ozone has shown equal or slightly better results. Thus as an alternative management strategy ozonated water which has fewer side effects can be considered a powerful tool in periodontal therapy.

\section{CONCLUSION}

Despite the substantivity of chlorhexidine, ozonated water shows slightly better results. Therefore the use of ozone is highly recommended as a potent atraumatic, antimicrobial agent in-home care and professional practice to treat periodontal disease non-surgically. The results obtained present a valid premise for further studies with larger sample size and microbiological parameters. 


\section{REFERENCES}

1. Brownstein $\mathrm{CN}$, Briggs SD, Schweitzer KL, et al. Irrigation with chlorhexidine to resolve naturally occurring gingivitis. A methodologic study. J Clin Periodontol. 1990 Sep; 17(8): 588-593.

2. Hardy JH, Newman HN, Strahan JD. Direct irrigation and subgingival plaque. J Clin Periodontol. 1982 Jan;9(1):57-65.

3. Slots J. Research, Science and Therapy Committee. Systemic antibiotics in periodontics. J Periodontol. 2004 Nov; 75(11):1553-1565.

4. Walsh TF, Glenwright HD, Hull PS. Clinical effects of pulsed oral irrigation with $0.2 \%$ chlorehexidine digluconate in patients with adult periodontitis. J Clin Periodontol. 1992 Apr; 19(4):245-248.

5. Kshitish D, Laxman VK. The use of ozonated water and $0.2 \%$ chlorhexidine in the treatment of periodontitis patients: a clinical and microbiologic study. Indian J Dent Res. 2010 JulSep; 21(3):341-348.

6. Gupta G, Mansi B. Ozone therapy in periodontics. J Med Life. 2012 Feb 22; 5(1):59-67.

7. Socransky SS, Haffajee AD. The bacterial etiology of destructive periodontal disease: current concepts. J Periodontol. 1992 Apr; 63(4 Suppl):322-331.

8. Goodson JM, Haffajee A, Socransky SS. Periodontal therapy by local delivery of tetracycline. J Clin Periodontol. 1979; 6:83-92.

9. Rams TE, Slots J. Local delivery of antimicrobial agents in the periodontal pocket. Periodontol 2000. 1996 Feb; 10: 139-59.

10. Chapple IL, Walmsley AD, Saxby MS, et al. Effect of subgingival irrigation with Chlorhexidine during ultrasonic scaling. J Periodontol. 1992 Oct;63(10):812-816.

11. Flemmig TF, Newman MG, Doherty FM, et al. Supragingival Irrigation with $0.06 \%$ Chlorhexidine in Naturally Occurring Gingivitis I. 6 Month Clinical Observations. J Periodontol. 1990 Feb; 61(2):112-117.

12. Estrela C, Estrela CR, Decurcio DA, et al. Antimicrobial efficacy of ozonated water, gaseous ozone, sodium hypochlorite and chlorhexidine in infected human root canals. Int Endod J.2007 Feb;40(2):85-93.

13. Huth KC, Saugel B, Jakob FM, et al. Effect of aqueous ozone on the NF-kappa B system. J Dent Res. 2007 May; 86(5):451-6.

14. Das S. Application of Ozone Therapy in Dentistry. IJDA. 3(2), April-June, 2011.
15. Huth KC, Quirling M, Lenzke S, et al. Effectiveness of ozone against periodontal pathogenic microorganisms. Eur J Oral Sci. 2011 Jun; 119(3):204-210.

16. Asari AM, Newman HN, Wilson M, et al. $0.1 \% / 0.2 \%$ commercial chlorhexidine solutions as subgingival irrigants in chronic periodontitis. J Clin Periodontol.1996 Apr; 23(4):320-325.

17. Dodwad V, Gupta S, Kumar K, et al. Changing paradigm in pocket therapy- ozone $v s$ conventional irrigation. Int $\mathrm{J}$ of Public Health Dent 2011:2(2):7-12.

18. Hujoel PP, DeRouen TA.Validity issues in split-mouth trials. J Clin Periodontol. 1992 Oct; 19(9 Pt 1):625-627.

19. Azmak N, Atilla G, Luoto $H$, et al. The effect of subgingival controlled-release delivery of chlorhexidine chip on clinical parameters and matrix metalloproteinase- 8 levels in gingival crevicular fluid. J Periodontol. 2002 Jun; 73(6):608-615.

20. Taggart JA, Palmer RM, Wilson RF. A clinical and microbiological comparison of the effects of water and $0.02 \%$ chlorhexidine as coolants during ultrasonic scaling and root planing. J Clin Periodontol. 1990 Jan; 17(1):32-37.

21. Christersson LA, Norderyd OM, Puchalsky CS. Topical application of tetracycline- $\mathrm{HCl}$ in human periodontitis. J Clin Periodontol. 1993 Feb; 20(2):88-95.

22. Stabholz A, Nicholas AA, Zimmerman GJ, et al. Clinical and antimicrobial effects of a single episode of subgingival irrigation with tetracycline $\mathrm{HCl}$ or chlorhexidine in deep periodontal pockets. J Clin Periodontol. 1998 Oct; 25(10):794-800.

23. Kalaga A, Addy M, Hunter B. Comparison of chlorhexidine delivery by mouthwash and spray on plaque accumulation. J Periodontol. 1989 Mar;60(3):127-30.

24. Ramzy MI, Gomaa HE, Mostafa MI, et al. Management of Aggressive Periodontitis Using Ozonized Water. Egypt. Med. J. N R C, 2005;6(1):229-245.

25. Nagayoshi M, Fukuizumi T, Kitamura C, et al. Efficacy of ozone on survival and permeability of oral microorganisms. Oral Microbiol Immunol. 2004 Aug; 19(4):240-246.

26. Huth KC, Jakob FM, Saugel B, et al. Effects of ozone on oral cells compared with established antimicrobials. Eur J Oral Sci. 2006 Oct;114(5):435-440.

27. Bassetti C, Kallenberger A. Influence of chlohexidine rinsing on the healing of oral mucosa and osseous lesions. J Clin Periodontol. 1980 Dec; 7(6):443-456.

28. Cline NV, Layman DL. The effects of chlorhexidine on the attachment and growth of cultured human periodontal cells. J Periodontal. 1992 July;598-602. 